\title{
MAIN RESULTS OF NUCLEAR DANGER EXAMINATION OF THE "SHELTER" OBJECT
}

\author{
V.I.Kupnyi, E.L.Belousov, A.S.Tovstogan (IA Chornobyl NPP)
}

Received December 10, 1997

\section{Background}

The "Shelter of the $4^{\text {th }}$ Unit of the Chernobyl Nuclear Power Plant" object belongs to the category of nuclear dangerous objects. The main source of danger in the "Shelter" object (SO) is considerable concentration of fuel-containing materials (FCM) which appeared as a result of the nuclear accident of 26 April, 1986. Nuclear danger of this concentration is caused by the existence of a potential possibility (risk) of the beginning of the self-supporting chain reaction of fission (SCR).

It is known that the reaction of the ${ }^{235} \mathrm{U}$ and ${ }^{239} \mathrm{Pu}$ fission has no lower limit and occurs during an interaction with any energy neutrons. There are always free neutrons in the fuel-containing medium. Their natural sources are cosmic radiation, spontaneous fission, as well as photo-neutron and $(\alpha, n)$ reactions. In the reaction of forced neutron-induced fission, instead of one neutron used for the fission, 2-3 new neutrons are released (the average output for ${ }^{235} \mathrm{U}-\sim 2.43$ neutron/fis., for ${ }^{239} \mathrm{Pu}-\sim 2.89$ neutron/fis.). It entails multiplication of the initial quantity of neutrons which can continue the chain of fission. Up to now the process of forced fission has been of a damping character, the multiplication property of the system can be characterized by the multiplication coefficient $\mathrm{M}$ :

$\mathrm{M}=\mathrm{S} / \mathrm{S}_{\text {source }}$, where: $\mathrm{S}_{\text {source }}$ (neutron/s) is a power of an independent neutron source; $\mathrm{S}$ (neutron/s) is a stabilized neutron power of the multiplication system, taking into consideration an additional inflow of neutrons due to the reaction of forced fission of nuclear fuel.

In contrast to spontaneous nuclear transformations, the intensity of which is stable and determined by the only law of radioactive decay, intensity of the reaction of forced neutron-induced fission changes, depending on the multiplication properties of a fuel-containing composition.

The index of the multiplication properties of the medium is the multiplication coefficient $\mathrm{K}_{\infty}$ characterizing the balance of neutrons in the reaction of forced fission at unlimited sizes of systems. If $\mathrm{K}_{\infty} \geq 1$ - the criticality becomes possible, i.e. reaching the level of neutrons reproducibility in the system by means of the reaction itself, sufficient for continuing forced fission of nuclear fuel without an inflow of neutrons from any other sources.

In a critical state the multiplication coefficient $M=\infty$ and neutron power may increase unlimitedly by means of the self-supporting chain reaction of fission. 
Subcriticality reserve is generally evaluated by the value that is inverse to the multiplication coefficient - subcriticality level $\delta \mathrm{K}_{\mathrm{ef}}=1 / \mathrm{M}$.

Subcriticality $\delta K_{\mathrm{ef}}=1-\mathrm{K}_{\mathrm{ef}}\left(\mathrm{K}_{\mathrm{ef}}<1\right)$, where $\mathrm{K}_{\mathrm{ef}}$ - effective coefficient of neutron multiplication in the system of limited sizes.

$\mathrm{K}_{\mathrm{ef}}$ is used to characterize both subcritical $\left(\mathrm{K}_{\mathrm{ef}}<1\right)$, and supercritical systems $\left(\mathrm{K}_{\mathrm{ef}}>1\right)$. If $\mathrm{K}_{\mathrm{ef}}=1$, then the system is in a critical state, i.e. the process of forced neutron-induced fission becomes undamping. The level of deviation of the multiplication system state from the critical state is characterized by the reactivity value $\rho$ :

$$
\rho=\frac{K_{e f}-1}{K_{e f}} \bullet 100 \%
$$

At the positive reactivity $\rho>0,5 \%$ the period of the neutron power doubling is reduced to the fractions of a second which makes the chain reaction of fission virtually uncontrollable and entails a nuclear accident with grave radioactive consequences. In 1986 the chain nuclear reaction inside the $4^{\text {th }}$ reactor of the ChNPP was stopped by the total self-destruction of the active zone. But a large quantity of the nuclear fuel that remained inside the $4^{\text {th }}$ unit (almost 200 tons with the effective enrichment $>1 \%$ for ${ }^{235} \mathrm{U}$ ) still keeps the threat of a new formation of local critical masses and appearance of a self-supporting chain reaction (i.e. the secondary nuclear accident). It is not excluded that such critical compositions could have spontaneously appeared during the active phase of the accident and have been one of the causes of the intensification of accidental ejection of radioactivity from the $4^{\text {th }}$ unit in the period from 1 May to 5 May, 1986. At the same time the processes of graphite burn-out, changing the fuel configuration, its mixing with destroyed construction materials were conducive to the transition of nuclear fuel to the subcritical state $K_{\mathrm{ef}}<1$ and to the cessation of the active phase of the accident.

As for spontaneously formed concentrations of fuel containing materials in the $4^{\text {th }}$ unit, the absolute level of subcriticality $\delta K_{e f}=1-K_{e f}\left(K_{e f}<1\right)$ is not guaranteed. In the context of nuclear safety the "Shelter" object represents a spatially scattered uncontrolled concentration of nuclear dangerous fissionable materials without any means of the active influence on criticality.

\section{Results of the studies in 1986-1990}

Experimental and computed data on the nuclear safety of fuel-containing masses (FCM) of the "Shelter" object, collected by the mid 1990, were summarized in the "Feasibility Study of the Nuclear Safety of the "Shelter" Object" (FSNS), published in 1990 by the Institute of Atomic Energy named after I.V.Kurchatov [1].

Calculations of neutron-physical characteristics of the FCM demonstrated that a potential threat comes from mixtures of FCM with water. Water is an 
effective moderator of fast neutrons to heat energy. In the sphere of low energy the possibility of a neutron fission reaction for ${ }^{235} \mathrm{U}$ and ${ }^{239} \mathrm{Pu}$ increases hundreds times and, correspondingly, the multiplication properties of fuel-containing compositions are changing. With the reduction (moderation) of the neutrons energy the possibility of a neutron fission reaction increases from $1.5-2$ barn (for fast neutrons) to 500 - 700 barn (for slow neutrons).

The maximal value of the multiplication coefficient $\mathrm{K}_{\infty}$ is achieved at a certain ratio of fuel and water which is called an optimum humidification. The form, dimensions and weight of the system, during which the condition of criticality $\mathrm{K}_{\mathrm{ef}}=1$ is achieved, are called critical ones. Multi-parametric calculations of neutron-physical parameters of homogeneous mixtures of lava-like FCM with water allowed us to determine the ratio between burning-out and mass share of $\mathrm{UO}_{2}$ in the mixture, which makes possible the existence of critical mass for optimum humidified LFCM (table 1). The value of dry mixture density $\left(\mathrm{SiO}_{2}\right.$ $+\mathrm{UO}_{2}$ ) was accepted to be equal to $2.5 \mathrm{gr} / \mathrm{cm}^{3}$.

Table 1. The values of burning-out and the $\mathrm{UO}_{2}$ share at which $\mathrm{K}_{\infty}=1$ for moist LFCM.

\begin{tabular}{|l|c|c|c|c|c|c|c|}
\hline Burning-out, $\mathrm{MW} \bullet$ day $/ \mathrm{kgU}$ & 0 & 2 & 4 & 6 & 8 & 10 & 12 \\
\hline Mass share of $\mathrm{UO}_{2}, \%$ & 20 & 25 & 29 & 34 & 38 & 43 & 50 \\
\hline
\end{tabular}

Figure 1.

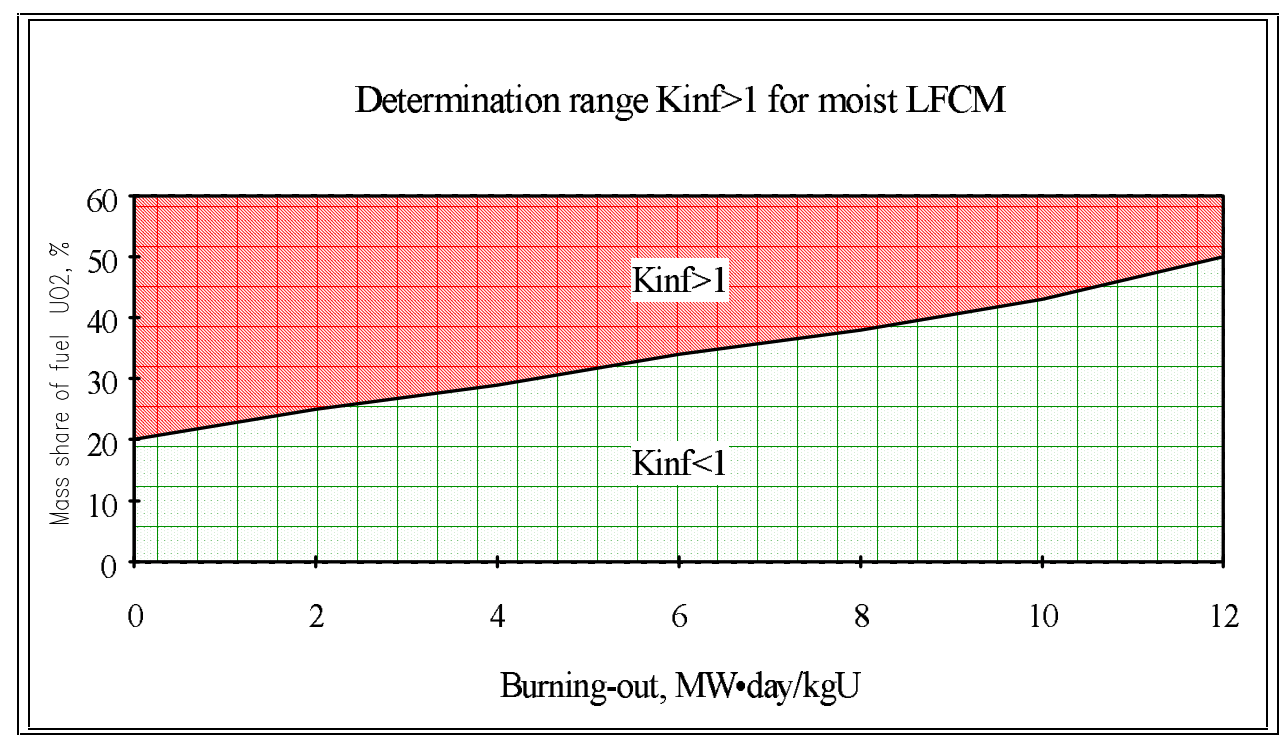

Calculations were performed for infinite homogeneous mixtures by means of the WIMS program, developed in Great Britain. It contains its own set of constants for 101 nuclides. The calculation of the neutrons spectrum in the WIMS program was carried out for 69 groups in the range of energy from 0 to $10 \mathrm{MeV}$. 
As for the FCM within the range $\mathrm{K}_{\infty}>1$ (picture 1), the calculation of the critical masses parameters were performed. Table 2 shows dependence of the minimum fuel critical mass of mixed burning-out (between 9 and 10 $\mathrm{MW} \bullet$ day $/ \mathrm{kgU}$ ) on the mass share of $\mathrm{UO}_{2}$ in the mixture with $\mathrm{SiO}_{2}$, when the density is $2,5 \mathrm{~g} / \mathrm{cm}^{3}$ and water concentration is optimum (without a reflector) [1].

Table 2. Critical parameters of homogeneous multiplication compositions $\left(\mathrm{UO}_{2}+\mathrm{SiO}_{2}+\mathrm{H}_{2} \mathrm{O}\right.$ with density $\left.\gamma_{(\mathrm{UO} 2+\mathrm{SiO} 2)}=2.5 \mathrm{~g} / \mathrm{cm}^{3}\right)$ of a spherical form.

\begin{tabular}{|l|c|c|c|c|c|c|c|c|}
\hline $\begin{array}{l}\text { Mass share of fuel } \\
\text { in the mixture, } \\
(\%)\end{array}$ & 40 & 41 & 42 & 43 & 46 & 49 & 50 & 55 \\
\hline $\begin{array}{l}\text { Partial density of } \\
\text { fuel, }\left(\mathrm{t} / \mathrm{m}^{3}\right)\end{array}$ & 1.0 & 1.025 & 1.05 & 1.075 & 1.15 & 1.225 & 1.25 & 1.375 \\
\hline $\begin{array}{l}\text { Partial density of } \\
\text { water, }\left(\mathrm{t} / \mathrm{m}^{3}\right)\end{array}$ & 0.24 & 0.25 & 0.26 & 0.26 & 0.29 & 0.31 & 0.33 & 0.38 \\
\hline $\begin{array}{l}\text { Multiplication } \\
\text { coefficient } \mathrm{K}_{\infty}\end{array}$ & 1.009 & 1.016 & 1.019 & 1.022 & 1.031 & 1.039 & 1.04 & 1.049 \\
\hline $\begin{array}{l}\text { Radius of a } \\
\text { critical sphere } \\
\text { (cm) }\end{array}$ & 331.7 & 259.7 & 231.7 & 211.6 & 170.6 & 146.5 & 139.8 & 116.6 \\
\hline $\begin{array}{l}\text { Critical mass of } \\
\text { the fuel } \mathrm{UO}_{2,} \mathrm{t}\end{array}$ & 152.9 & 75.2 & 54.6 & 42.6 & 23.9 & 16.1 & 14.3 & 9.13 \\
\hline
\end{tabular}

FSNS Conclusions:

"After the end of the active phase of the accident, a set of diagnostic measurements has been pointing out at subcriticality of all the fuel-containing materials in the "Shelter" object".

Quantitative indices of the multiplication coefficient obtained experimentally on the basis of special passive neutron methods, for all the FCM are less than 0.4, and on the basis of active methods - less than the sensitivity limit (0.7).

The calculations confirmed that all the FCM are in a deep subcritical state.

Various natural calamity events which cause the shifting of construction fragments of the destroyed unit (without water flooding), do not entail the appearance of critical systems.

Water can be the main factor causing the reduction of subcriticality of the FCM in the "Shelter" in the course of time".

\section{Results of the 1991-1996 examinations}

It should be noted that conclusions in the Feasibility Study of Nuclear Safety (FSNS) were based on the examinations carried out on the surface of fuel lava concentrations, since there was no technology for the extraction of highly active 
cores to penetrate into the lava. During the last few years (after the publication of FSNS) numerous experimental and computing examinations were performed for the determination of nuclear-physical, mechanical physico-chemical characteristics of the FCM related directly to the nuclear safety of the "Shelter" object. Below we list the most essential findings which resulted in a need for a substantial addition to the FSNS conclusions.

1) While earlier the penetration of water into lava-like FCM was impeded by its high temperature and water resistance of the matter, now the conditions have changed. Calculations and experiments reveal considerable cooling of the lava, its cracking and transformation into water-tight structure.

2) Examination of the fuel-containing lava samples from the premises $305 / 2$ (under the control room), carried out in 1992 - 1993, showed that some samples contained AZ (active zone) fragments in a non-melted form. Besides, visual observations showed zone fragments which were directly adjoining the lava. Thus, for calculations and assessment of the nuclear safety it is necessary to take into consideration the new composition "Lava + AZF + water" which is more dangerous than the "Lava + water" composition.

Preliminary calculations of critical parameters for such mixtures were performed in the "Kurchatov Institute" [2].

\section{Initial data:}

Isotope composition of the fuel:

$1 \%$ Uranium-235, 0.2\% Plutonium-239 (burning-out 10MW • day $/ \mathrm{kgU}$ )

Cell composition:

RBMK Fuel Elements (FE), water, LFCM.

LFCM composition:

Uranium dioxide $-0.2 \mathrm{~g} / \mathrm{cm}^{3}$

Silica dioxide $-2 \mathrm{~g} / \mathrm{cm}^{3}$

Reflector:

Concrete, $50 \mathrm{~cm}$.

Water content: $\quad$ chosen under condition $\mathrm{K}_{\infty}$ - $\max$.

The following data were obtained for the sphere geometry (table 3 ):

Table 3. Critical parameters for the mixture "lava + active zone fragments + water" with a concrete reflector.

\begin{tabular}{|c|c|c|c|c|c|}
\hline $\begin{array}{c}\mathrm{Vfcm} / \\
\mathrm{Vfe}\end{array}$ & $\begin{array}{c}\text { density } \mathrm{H}_{2} \mathrm{O}, \\
\text { volumetr. } \%\end{array}$ & $\begin{array}{c}\text { Coefficient } \\
\mathrm{K}_{\infty}\end{array}$ & $\begin{array}{c}\text { R cr. sph. } \\
\mathrm{cm}\end{array}$ & \multicolumn{2}{|c|}{$\mathrm{UO}_{2}$, ton } \\
\hline 0 & 60 & 1.17 & 36.5 & 0.8 & 0 \\
\hline 1.26 & 37 & 1.15 & 51 & 1.34 & 0.04 \\
\hline 2.75 & 27.5 & 1.13 & 66 & 2.14 & 0.12 \\
\hline 4.50 & 21.0 & 1.11 & 84 & 3.20 & 0.30 \\
\hline 6.40 & 17.2 & 1.09 & 110 & 6.00 & 0.80 \\
\hline 8.60 & 13.8 & 1.07 & 145 & 11.0 & 2.00 \\
\hline
\end{tabular}

At fuel enrichment $2 \%$ for Uranium-235 (fuel with low burning-out) the radius of critical spheres reduces two times.

Assessment calculations for analogous compositions were also performed in SSC RF PEI [6]. Previously verified complex REDUT was used as a software. 
Table 4 shows results of calculations of critical parameters for an equally sized cylinder $(\mathrm{H}=\mathrm{D})$ with a $50 \mathrm{~cm}$. thick concrete reflector.

Table 4. Critical parameters for homogeneous-heterogeneous composition of fuel tablets, LFCM and water at burning - out $10.8 \mathrm{MW} \bullet$ day $/ \mathrm{kgU}$.

\begin{tabular}{|c|c|c|c|c|c|}
\hline$\#$ & $\begin{array}{c}\text { Volumetric } \\
\text { share of LFCM } \\
\%\end{array}$ & $\begin{array}{c}\text { Volumetric } \\
\text { share of } \mathrm{H}_{2} \mathrm{O}, \\
\%\end{array}$ & $\begin{array}{c}\text { Critical } \\
\text { volume, } \mathrm{L}\end{array}$ & $\begin{array}{c}\text { Weight of } \mathrm{UO}_{2} \\
\text { in LFCM, } \\
\mathrm{t}\end{array}$ & $\begin{array}{c}\text { Weight of } \mathrm{UO}_{2} \\
\text { in tablets, } \\
\mathrm{t}\end{array}$ \\
\hline 1 & 90 & 10 & \multicolumn{3}{|c|}{$\mathrm{K}_{\mathrm{ef}}=0,993$} \\
\hline 2 & 85 & 15 & 16148 & 2.24 & 29.5 \\
\hline 3 & 80 & 20 & 4890 & 0.639 & 8.9 \\
\hline 4 & 75 & 25 & 2756 & 0.338 & 5.0 \\
\hline 5 & 60 & 40 & 1100 & 0.108 & 2.0 \\
\hline 6 & 40 & 60 & 785 & 0.051 & 1.4 \\
\hline
\end{tabular}

When there is no water, the system remains deeply subcritical under any conditions. But, as it has been pointed out, in practice there are no working barriers for water inflow.

Taking the following facts into consideration:

- The considerable lowering of the barriers which used to present the selfsupporting chain reaction;

- Detection of new, potentially dangerous compositions of FCM;

- Classification (based on a conservative approach) of the "Shelter" premises according to the level of nuclear danger and a number of other reasons -

we can arrive at the conclusion that:

at present, in some premises of the "Shelter" object and with respect to those FCM concentrations about which there is no sufficient information, under certain initial conditions there exists a possibility for the appearance of FCM criticality.

Such premises include the Central Room, where dozens of tons of nuclear fuel may be under the layer of thrown-off materials, the reactor pit, the premises under the control room where the most part of the fuel "lava" is located.

It should be noted that until recently some abnormal phenomena were registered related to the increase in the density of a neutron flux which may have been caused by both the change in the multiplication properties of fuel masses due to water inflow, and the increase in the instrumental error - due to the same reason.

\section{Assessment of the self-supporting chain reaction consequences on the premises of the "Shelter" object $[5,6]$}

At present, the most dangerous scenario of the development of the local selfsupporting chain reaction (SCR) is connected with a fast increase of radioactivity when flooding the fuel-containing masses with water. Without protection barriers, 
the consequences of such an event will be irradiation of the "Shelter" object personnel with a powerful penetrating neutron $\gamma$-radiation.

Duration of the neutron burst during the SCR $-0.3 \mathrm{~s}$, energy release $-10^{9} \mathrm{~J}$, fission number $-3 \cdot 10^{1 \cdot .9}$. At this number of fissions, about $4 \mathrm{kKi}$ of radioactive inert gases (RIG) and radionuclides of iodine are generated. The consequences of their release into the atmosphere will be irradiation of the personnel nearby the "Shelter" object with up to 1 rem doses [2].

As to the personnel working inside the "Shelter" object, criticality of the FCM may result in irradiation with greater doses.

Density of the prompt neutron flux in the zone of SCR development is assessed $\sim 10^{16} \mathrm{n} / \mathrm{cm}^{2} \cdot \mathrm{s}$, and at the distance of $20 \mathrm{~m}-10^{12} \mathrm{n} / \mathrm{cm}^{2} \cdot \mathrm{s}$. The expected dose of neutron irradiation in the zone of SCR development is assessed to be at the level of $\sim 10^{7} \mathrm{rem}$, and at the distance of $20 \mathrm{~m}-10^{4} \mathrm{rem}$.

The exposed dose rate of a prompt $\gamma$-radiation in the zone of SCR development is assessed as the value equal to $\sim 10^{6} \mathrm{R} / \mathrm{s}$, and at the distance of $20 \mathrm{~m} \sim 300 \mathrm{R} / \mathrm{s}$, at the same time $\gamma$-doses during the SCR $(0.3 \mathrm{~s})$ at these points (due to prompt $\gamma$-quantum) may be equal to $\sim 10^{6} \mathrm{rem}$ and $100 \mathrm{rem}$, correspondingly.

As long as several hours will take to decrease the dose rate of $\gamma$-radiation from fission products (formed during the SCR) from $300 \mathrm{R} / \mathrm{s}$ down to the level commensurable to background values at the FCM surface $(800 \div 80 \mathrm{R} / \mathrm{h})$.

Assessment of the explosion energy that may happen in case of the SCR shows that with the existing geometry of FCM, the kinetic energy of scattering the critical mass fragments will not exceed 0.5 MJ. The trinitrotoluene equivalent of this value is no more than $0.5 \mathrm{~kg}$ of the explosive. Such an explosion is dangerous not because of the possibility of destroying building constructions, but because of the possibility of releasing radioactive dust and aerosol from inside the "Shelter" object into the environment. Water evaporation during the SCR may also cause formation of aerosols and result in the additional release of radioactivity into the atmosphere.

\section{First priority measures for the "Shelter" object water management}

Water is not only a factor of the potential nuclear danger of FCM, but it also determines the radio-and- ecological danger of the "Shelter" object in the longterm perspective. Different projects, elaborated at present for the object modernization, are called upon, first of all, to prevent the contact of the FCM with water.

In parallel, in order to avoid radioactive leakage into the soil, it is necessary to solve the problem of collection, removal and treatment of the water accumulated in the northern and central parts of the "Shelter" object. 
Water chaotically accumulates in the reactor sector above the level of ground water, that is why there is a possibility of uncontrolled migration of radioactivity beyond the "Shelter" limits.

There are about $500 \mathrm{~m}^{3}$ of water in room $001 / 3$ of the ASRC, that runs from the reactor sector. Up to $200 \mathrm{~m}^{3}$ of water may accumulate in the premises of lower points of the reactor sector as a result of atmospheric precipitation and water from dust suppression. As for the level of activity - from $4.0 \times 10^{-6}$ to $1.4 \times 10^{-2} \mathrm{Ki} / 1$ - the water accumulations in the premises of the reactor sector are regarded as liquid radioactive wastes of the medium activity (LRW). Radionuclide composition of the samples according to gamma-spectroscopy is: Cs-137-98-99\%; Cs-134-1-2\%. Activity of the examined water samples as to the $\beta$-radiation isotope - Strontium- 90 - is $2.6 \times 10^{2} \div 7.9 \times 10^{6} \mathrm{~Bq} / 1\left(7 \times 10^{-9} \div\right.$ $\left.2.1 \times 10^{-4} \mathrm{Ki} / 1\right)[3]$.

The water is washing out the FCM, that is why, until recently, besides Cesium and Strontium isotopes, there was a rise of concentration of alpha-active and fissionable isotopes of Uranium (up to $60 \mathrm{mg} / \mathrm{l}$ ) and Plutonium ( 3 kBq/l)[3]. Presence of highly concentrated U-containing water in the reactor section of the $4^{\text {th }}$ unit is considered to be the principal contradiction against the discharge of LRW of the medium activity from the "Shelter" object to the system of LRW treatment of operating ChNPP units, since it may disturb the water mode and equipment operation in the system of LRW treatment. At present, the "Shelter" object lacks the system of pumping-out and treatment of such water. Development of technology for the treatment of U-containing LRW of the medium activity is still at the stage of scientific research.

Complex solution of the problem concerning the water inside the "Shelter" object, was envisaged within the framework of international cooperation [9]. This task was included in the list of short-term measures (STM 12) developed by the International Expert Group within the TACIS project on nuclear safety "ChNPP Unit 4, Short and Long Term Measures". "The Recommended Course of Actions" developed on the basis of the system analysis defines a frame concept including a series of steps aimed at the reduction of the risk level, nuclear and radioactive danger and at bringing the "Shelter" object to the environmentally safe condition.

The comprehensive "Shelter Implementation Plan" (SIP) was developed in cooperation with the EC, USA, Ukraine and a group of international experts in order to ensure a detailed work plan for the implementation of these recommendations. Short- and long-term measures formulated earlier in the "Recommended Course of Actions" were included in the detailed "Work Breakdown Structure" of the integrated plan, containing 22 tasks. On the basis of a deep study of work the SIP defined some projects requiring urgent implementation. These projects pertain to the first part of Task 13 (WBS 1.3.02) concerning water management and including one of the main program stages of SIP - decision on the system of water management (P6).

It was decided to hold an international tender for all engineering and designing works related to the system of water management on order to deal with the water contained in the "Shelter". Tendering for works on the Shelter Project 
will be conducted in compliance with the "Procurement Policies and Rules for the Projects Financed by the EBRD" and any special instructions issued by the EBRD for implementing the SIP.

The total cost of first priority measures within the $13^{\text {th }}$ Task (WBS 1.3.02.03 - 1.3.02.30) is estimated in the amount of $\$ 1833000$. To reduce the impact of the aqueous medium of the "Shelter" object on its safety and environment it is envisaged to determine the sources, water migration routes, quantity of radioactive and fissionable materials contained in the water of the "Shelter" object. The plan of water management should be developed and implemented, which would include specification of the water collection points, determination of the strategy of the control and the monitoring of water, as well as technological schemes of pumping-out and the purification of water.

The first stage of the work will be completed in 1998, after the final approval of the conceptual design and principal consent of the regulatory body.

\section{Nuclear safety during the treatment of the "Shelter" water}

In order to facilitate the designing of installations and selection of equipment, meeting the requirements of nuclear safety, scientific and research work was carried out in 1996 for the purpose of nuclear and radioactive safety during the management of water (LRW) containing fissionable materials [7]. Below is given an assessment of minimum critical and safe parameters for the most dangerous case - a mixture of Uranium dioxide of $2 \%$ enrichment with water (table 5).

Table 5. Values of minimum critical and safe parameters for the homogeneous mixture of $\mathrm{UO}_{2}(2 \%$ enrichment) with water. Reflector - water.

\begin{tabular}{|l|c|c|c|}
\hline Parameter & Critical value & Safe value & Optimal $\mathrm{C}_{\mathrm{U}}, \mathrm{g} / \mathrm{l}$ \\
\hline $\begin{array}{l}\text { Max. concentration of Uranium } \\
\mathrm{g} / \mathrm{l}\end{array}$ & 800 & 615 & - \\
\hline Weight of Uranium, kg & 220 & 104 & 2000 \\
\hline Volume of sphere, $\mathrm{l}$ & 95 & 73 & 2700 \\
\hline Diameter of infinite cylinder, cm & 40 & 36 & 2700 \\
\hline $\begin{array}{l}\text { Thickness of infinite layer (plate), } \\
\text { cm }\end{array}$ & 22 & 20 & 2800 \\
\hline Max. water content, mass \% & 4 & 3 & \\
\hline
\end{tabular}

Specified values of parameters will be regarded as outdated. The assessment of nuclear safety of individual equipment requires data and information on the processes parameters.

Due to the possible formation of critical masses out of new formations the calculations were carried out for the assessment of critical parameters of water composition systems on the basis of salt $\mathrm{Na}_{4} \mathrm{UO}_{2}\left(\mathrm{CO}_{3}\right)_{3}$. These assessments, as well as the known experimental data on criticality of other Uranium compounds, show that minimum critical parameters of the systems with low-enrichment 
Uranium may be realized in the range of values of the moderation parameters $\mathrm{H} / \mathrm{U}$ about 500 . It corresponds (with $2 \%$ enrichment) to $\mathrm{H} / \mathrm{U}=10$ (water content is about $13 \%$ ), and minimum values of critical parameters are: $\mathrm{R}_{\mathrm{cr}}=81.7 \pm 2 \mathrm{~cm}$; $\mathrm{V}_{\mathrm{cr}}=2287 \pm 160 \mathrm{l} ; \mathrm{G}_{\mathrm{cr}}^{\mathrm{U}}=2414 \pm 170 \mathrm{~kg}$. The assessment error corresponds to \pm $0.7 \%$ in $\mathrm{K}_{\text {ef. }}$ Calculations were carried out by means of PC CRAB, while table values contain corrections, taking into account the results of their verification according to the data of critical experiments $[7,8]$

Assessment of the consequences of the SCR in the system consisting of new formations (salt $\mathrm{Na}_{4} \mathrm{UO}_{2}\left(\mathrm{CO}_{3}\right)_{3}$ ) gave the following results [8]:

- the total number of fissions during the SCR is $1.6 \times 10^{19}$ fission;

- the total outburst of volatile radionuclides (RIG and J) is $\sim 2000 \mathrm{Ki}$.

\title{
References
}

[1] Feasibility Study of the Nuclear Safety of the "Shelter" object. IAE n.a. Kurchatov, Moscow, 1990, p. 127.

[2] "State of Nuclear, Radiation, Ecological Safety of the "Shelter" object. (Collection, verification, submission of information, fulfillment of additional studies) S.A. Bogatov, A.A. Borovoy, S.A. Gavrilov and others. Report \#130-09/38 of 15.12.95, IVTEM RSC "Kurchatov Institute", Moscow, 1995.

[3] Research and diagnostic work at the "Shelter" object for provision of initial data to correct "Regulations". Stage 11. Analysis of obtained results and development of recommendations for improvement of radiation and ecological safety of SO. Report \#1-95/9-2 of 18.12.96. MSTC "Shelter" of the NAS of Ukraine, Chornobyl, 1996, p. 80.

[4] Expert conclusion on the materials about assessment and prognosis of the state of nuclear and radiation safety of the "Shelter" object. 23.06.96. PEI, Obninsk, 1992, p. 33.

[5] Development of scenario and methodology of assessment of SCR consequences during treatment of FCM penetration of water. Tech. Ref. \#30-05/42 of 14.06.96, PEI, Obninsk, 1996, p. 10.

[6] Development of scenario and methodology of assessment of SCR consequences during treatment of FCM penetration of water. Tech. Ref. \#29-28/33 of 27.06.96, PEI, Obninsk, 1996, p. 22.

[7] Development of quantitative indices of the NS level and requirements to NS during execution of work with LRW at SO and the $3^{\mathrm{d}}$ Unit of ChNPP. Tech. Ref. \#29-28/37 of 15.08.96, PEI, Obninsk, 1996, p. 14.

[8] Development of scenario and methodology of assessment of SCR consequences during treatment of LRW, containing NWLL at SO and the $3^{\mathrm{d}}$ Unit of ChNPP. Tech. Ref. \#29-28/32 of 27.06.96, PEI, Obninsk, 1996, p. 18.

[9] Chornobyl Unit 4. Shelter Implementation Plan. Tacis Services DG IA, European Commission, Brussels, 31 May 1997, p. 231.

\section{ОСНОВНІ РЕЗУЛЬТАТИ ВИВЧЕННЯ ЯДЕРНОЇ НЕБЕЗПЕКИ ОБ'СКТУ “УКРИТТЯ”}

\author{
В.І.Купний, Є.Л.Білоусов, А.С.Товстоган
}

